\title{
FAVOR (FAst Variability Optical Registration) - A two-telescope complex for detection and investigation of short optical $\operatorname{transients}(*)$
}

G. Beskin, V. Bad'in, A. Biryukov, S. Bondar, G. Chuntonov

V. Debur, E. Ivanov, S. Karpov, E. Katkova, V. Plokhotnichenko

A. Pozanenko, I. Zolotukhin, K. Hurley, E. Palazzi, N. Masetti, E. Pian

L. Nicastro, C. Bartolini, A. Guarnieri, A. Piccioni, P. Conconi

E. Molinari, F. M. Zerbi, N. Brosch, D. Eichler, A. Shearer, J.-L. Atteia and M. BOER

Special Astrophysical Observatory of RAS, Nizhnij Arkhyz - Karachai-Cherkessia Russia, 369167

(ricevuto il 23 Maggio 2005; pubblicato online il 23 Settembre 2005)

Summary. - An astronomical complex intended to detect optical transients (OTs) in a wide field and follow them up with high time resolution investigation is described.

PACS 95.55.Cs - Ground-based ultraviolet, optical and infrared telescopes.

PACS 01.30.Cc - Conference proceedings.

The nature of the Gamma-Ray Bursts (GRBs), the most powerful events in the Universe, remains mysterious despite many investigations in the last years. It is clear that their progenitors are extremely compact stellar objects, such as neutron stars (NS) or even black holes $(\mathrm{BH})$, either isolated or in binary systems. Their internal spatial structure and dynamics, and the processes transforming their energy into GRBs are inevitably reflected in the temporal properties of bursts. While the full duration of the GRBs covers a $0.01-100 \mathrm{~s}$ range, the light curves of the long ones show a substructure in $80 \%$ of the cases, and for $66 \%$ the time scales of the short ones variability are shorter than 0.1 of their duration [1]. Moreover, millisecond features are discovered in the light curves of some long-duration bursts [2]. At the same time, a number of models predict the generation of considerable optical fluxes simultaneous with the GRB, which can reach 8-12 magnitude [3-5]. This is especially important due to the fact that for reasonable spectral assumptions the amount of optical photons exceeds by factors of 100-1000 the number of gamma-ray photons. The results of a unique quasi-synchronous detection of a GRB optical transient (OT; the optical exposure was started $22 \mathrm{~s}$ after the trigger)

(*) Paper presented at the "4th Workshop on Gamma-Ray Burst in the Afterglow Era", Rome, October 18-22, 2004.

(C) Società Italiana di Fisica 


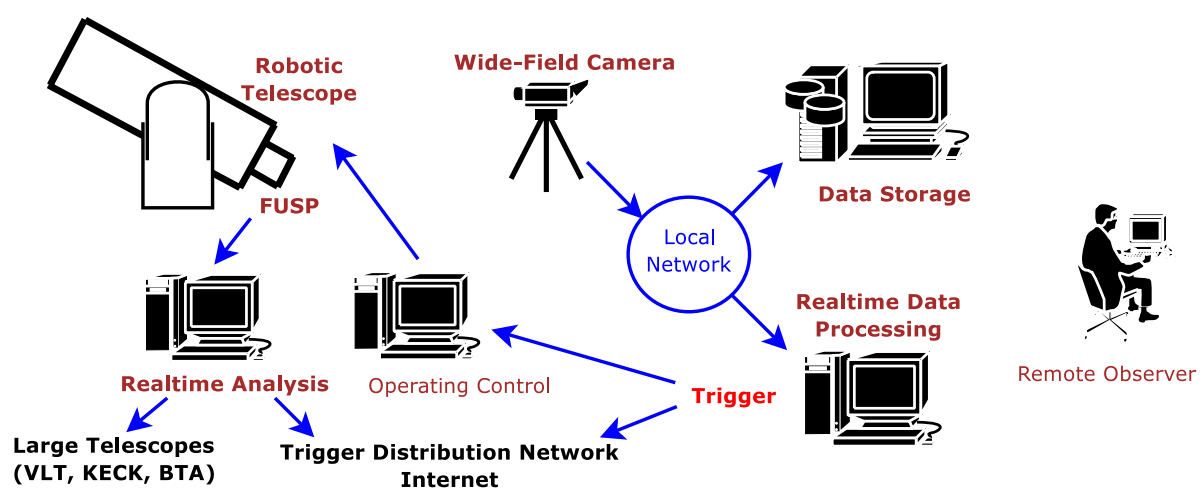

Fig. 1. - General scheme of the FAVOR complex.

confirm such a relation [6]. Therefore, the search for, the detection and the detailed high temporal resolution study of OTs accompanying GRBs may bring decisive information on the nature of this phenomenon.

It is clear that observations such as those described here must be carried out independently of triggers created by space-borne telescopes with instruments equipped with wide fields of view, comparable with those of satellite instruments $\left(>100 \mathrm{deg}^{2}\right)$. This approach has been realized in the creation of wide-field camera with high temporal resolution [7]. Note, however, that wide-field monitoring telescopes cannot have objectives with diameter large than 15-20 cm (due to the growth of optical aberrations) and thus may be used only for the detection of OTs as a source of triggers. These very wide field instruments must pass the OT information on to the mid-sized telescope (50-200 cm diameter) for detailed study. Two-telescope complexes must be created [8]. In some sense, terrestrial automatic optical telescopes receiving event triggers from space-borne gamma detectors form two-telescope complexes similar to those described above. However, due to time delays in the information transfer through global networks it has never been possible to start optical observations earlier than 20-60 s from the high-energy trigger. Moreover, even UVOT, the dedicated optical telescope on board the Swift satellite, starts observations only 30-100 s after the gamma-ray photon detection.

Taking these into account, a set of requirements for a two-telescope complex for the detection and investigation of fast optical transients could be formulated: formation of the trigger by the monitoring instrument in real time within $0.5-1 \mathrm{~s}$ from detection or faster; minimal time delay for data transfer to the second-level telescope (local networkless than $0.5 \mathrm{~s}$ ); minimal pointing time towards the OT (slew speed faster than 5-7 $\mathrm{deg} / \mathrm{s})-1-2 \mathrm{~s}$; maximal temporal resolution for both instruments-better than $0.5 \mathrm{~s}$ for the monitoring one, better than $10^{-3} \mathrm{~s}$ for the second-level telescope; extraction of maximum possible information - temporal, spatial, spectral and polarimetric - from each recorded source photon by the second telescope instrument. Therefore, this is conceived to be a panoramic spectropolarimeter; fully automatic operation of the complex.

We proposed to assemble such a research complex and began its development [8].

The complex (see fig. 1) consists of a Wide-Field Camera (WFC), a real-time data processing computer complex (RTDC) and a robotic telescope (RT) equipped with a Fast Universal Spectro-Polarimeter (FUSP).

The WFC [9] has a $15 \mathrm{~cm}$ objective with fast $\left(f_{\sharp}=1.2\right)$ focal ratio, which yields a wide field of view of $16^{\circ} \times 21^{\circ}$. It is equipped with a VS-CTT285-2001 TV-CCD camera 


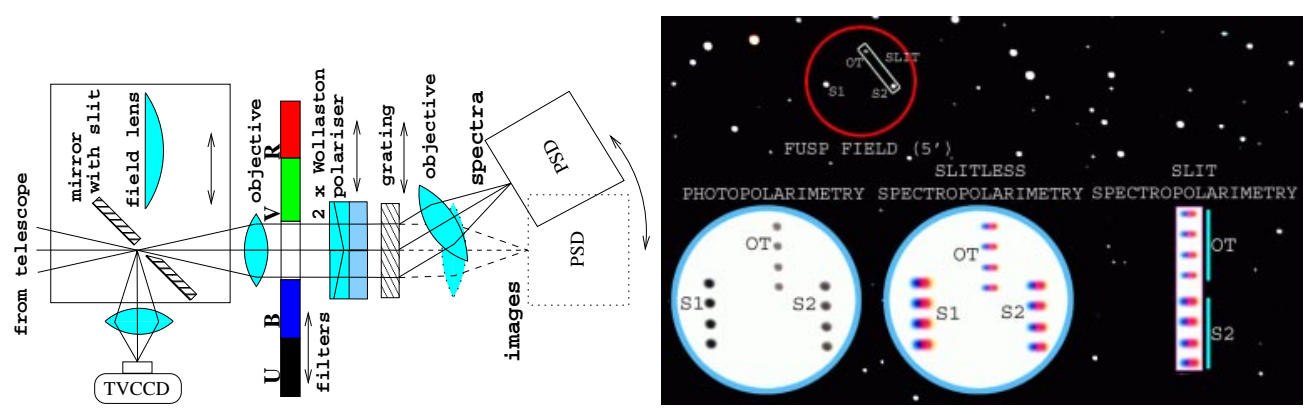

Fig. 2. - FUSP principal scheme (left panel) and sketch of its field of view in various modes (right panel).

$(1280 \times 10246.5$ microns pixels $)$ with a $7.5 \mathrm{~Hz}$ frame rate $(0.13 \mathrm{~s}$ exposure duration per frame). The data flow from the camera is processed on several time scales simultaneously. The shortest time scale is that of frame acquisition - the transient may be detected and classified if seen for $0.4 \mathrm{~s}-i . e$. on three successive frames (this aims to filter out the noise and moving objects such as satellites and meteors). The limiting magnitude on this time scale is roughly 11.5 in a spectral band close to V. On the second time scale, sums of 100 successive frames are examined; this leads to a $14^{m}$ limit with $13 \mathrm{~s}$ temporal resolution and allows the detection of slowly rising transients.

The WFC will operate in conjunction with a RT able to automatically slew to the position of detected OT for detailed studying. An example of such a telescope is the REM-Rapid Eye Mount [10], which has a $60 \mathrm{~cm}$ mirror with a $4.8 \mathrm{~m}$ effective focal length and is located at La Silla Observatory, in Chile. Currently it can slew at a $5^{\circ} \mathrm{s}^{-1}$ rate and is equipped with the REMIR near-infrared camera and ROSS (REM Optical Slitless Spectrograph) instrument, therefore it can perform detailed investigation of OTs on a time scale of a few seconds after the trigger reception.

Such a RT equipped with the FUSP will allow detailed investigations of OTs in spectropolarimetric mode and with high time resolution; this would maximize the collected information about each detected source photon.

The layout of FUSP is shown on the left panel of fig. 2. The device consists of an input unit, which allows the choice of three observing modes (photopolarimetry, slitless and slit spectropolarimetry), collimating optics, a polarization unit (a double Wollaston prism), a set of filters, a dispersion element (prism), a position-sensitive detector (PSD) with time resolution of 1 microsecond, and an acquisition system of type "QUANTOCHRON4-96" [11]. In order to control the observations, the FUSP is equipped with a TV-CCD guide.

In PHOTOPOLARIMETRIC mode, the field lens forms a 4-5 arcmin diameter image of the FOV in the collimator focal plane. The PSD is placed on the same axis with this lens, the collimator, the polarizer and one of the filters in the set (U, B, V, or R). The PSD records photons from each object in the FOV on four simultaneous images, with different polarization plane orientations. For an exposure time of one second it is possible to measure $30 \%$ linear polarization (3 Stokes parameters simultaneously) at a $3 \sigma$ level, for an object with $V \approx 13.5(S / N=10)$.

In SLITLESS SPECTROPOLARIMETRIC mode, the PSD is placed on the same axis with the prism and the collimator output lens. Images of four spectra with different polarization plane orientations, for each object in the FOV, are projected onto the PSD photocathode. A spectral range of $4000-7000 \AA$ is covered with a spectral resolution of 
100-250 A. Under moderate seeing and sky brightness conditions it is possible to measure a $10-15 \%$ linear polarization at a $3 \sigma$ level for an object with $V \approx 11.5-12(S / N=10)$.

In SLIT mode, a diagonal mirror with a slit size of $2^{\prime \prime} \times 4^{\prime}$ replaces the field lens, and the OT and its comparison star are projected onto the slit. Photons of their four spectra are recorded by the PSD. In this mode, detection limit is $V \approx 13-13.5^{m}$ for the conditions described above.

In order to change observing modes, a set of motors under computer control is used. An average duration for this mode change is a few seconds.

The detector to be used in FUSP is the new generation of Position-Sensitive Detector with high time resolution. This device will be able to record individual photons with spatial resolution of $10-20 \mu \mathrm{m}$, measure their arrival times with accuracy of 10 nanoseconds, and accumulate these data continuously without any gaps for several hours. Its quantum efficiency will be $20-35 \%$ in the $360-850 \mathrm{~nm}$ band while providing a large number of pixels $\left(10^{6}\right)$ and event rates up to $10^{5}$ counts per second.

Simulated results of FUSP observations in different modes are shown in the right panel of fig. 2. FUSP is now in its manufacturing stage, on the basis of the experience of development of Multichannel Panoramic Photometer-Polarimeter which now is in operation at the 6 -meter telescope of SAO RAS $[12,11]$.

The selection of observation modes is based on the brightness of the OT as measured by the WFC (on the last frame used for its classification) and the prediction of its brightness for the observations start time. The brightness of OTs may be measured on a single frame (0.13 s exposure) or on a sum of 100 frames (13 s); this yields detection limits in the $\mathrm{V}$ band of 11.5 and $14 \mathrm{mag}$, respectively.

The observation mode is selected during the pointing time $(1-3 \mathrm{~s})$ : for $V<11.5$ slitless spectropolarimetry, and for $V=11.5-14$ - photopolarimetry, which is followed by the slit spectropolarimetry after the determination of the OT position.

To conclude, FAVOR will be able to detect and study with high temporal resolution OTs as faint as $14^{m}$ in 1-60 s after their detection.

$$
* * *
$$

This work was supported by grants of CRDF (No. RP1-2394-MO-02), RFBR (No. 0402-17555), and INTAS (04-78-7366). SK thanks Russian Science Support Foundation for support. GB thanks the Cariplo Foundation for a scholarship, and Merate Observatory for hospitality.

\section{REFERENCES}

[1] Nakar E and Piran T., MNRAS, 330 (2002) 920.

[2] McBreen S. F. et al., A\&A, 380 (2001) L31.

[3] Liang et al., ApJL, 519 (1999) L21.

[4] Eichler D. and Beskin G., Phys. Rev. Lett., 85 (2000) 13.

[5] Wu et al., MNRAS, 342 (2003) 1131.

[6] Akerlof C. W., Nature, 398 (1999) 400.

[7] KARPOV S. et al., these proceedings.

[8] Karpov S. et al., Astron. Nach., 325 (2004) 677.

[9] Zolotukhin I. et al., Astron. Nach., 325 (2004) 675.

[10] Zerbi R. et al., Astron. Nach., 322 (2001) 275.

[11] Plokhotnichenko et al., Nucl. Instrum. Meth. A, 513 (2003) 167.

[12] Debur V. et al., Nucl. Instrum. Meth. A, 513 (2003) 127. 